\title{
The Process of Modeling Information System Architectures with IHE
}

\author{
Angela MERZWEILER ${ }^{\mathrm{a}, 1}$,Sebastian STÄUBERT ${ }^{\mathrm{b}}$, Alexander STRÜBING ${ }^{\mathrm{b}}$, \\ Armel TONMBIAK ${ }^{\mathrm{a}}$, Knut KAULKE ${ }^{\mathrm{c}}$, Johannes DREPPER ${ }^{\mathrm{c}}, \mathrm{Björn} \mathrm{BERGH}^{\mathrm{d}}$ \\ and Alfred WINTER ${ }^{\mathrm{b}}$ \\ a Department MIS, Universitätsklinikum Heidelberg \\ b Institute for Medical Informatics, Statistics and Epidemiology, Universität Leipzig \\ ${ }^{c}$ TMF e.V. \\ ${ }^{d}$ Institut für Medizinische Informatik, Universitätsklinikum Schleswig-Holstein
}

\begin{abstract}
IHE has defined more than 200 integration profiles in order to improve the interoperability of application systems in healthcare. These profiles describe how standards should be used in particular use cases. These profiles are very helpful but their correct use is challenging, if the user is not familiar to the specifications. Therefore, inexperienced modelers of information systems quickly lose track of existing IHE profiles. In addition, the users of these profiles are often not aware of rules that are defined within these profiles and of dependencies that exist between the profiles. There are also modelers that do not notice the differences between the implemented actors, because they do not know the optional capabilities of some actors. The aim of this paper is therefore to describe a concept how modelers of information systems can be supported in the selection and use of IHE profiles and how this concept was prototypically implemented in the "Three-layer Graph-based meta model" modeling tool $\left(3 \mathrm{LGM}^{2} \mathrm{Tool}\right)$. The described modeling process consists of the following steps that can be looped: defining the use case, choosing suitable integration profiles, choosing actors and their options and assigning them to application systems, checking for required actor groupings and modeling transactions. Most of these steps were implemented in the $3 \mathrm{LGM}^{2}$ Tool. Further implementation effort and evaluation of our approach by inexperienced users is needed. But after that our tool should be a valuable tool for modelers planning healthcare information system architectures, in particular those based on IHE.
\end{abstract}

Keywords. Health Information Systems, Hospital Information Systems, Health Information Interoperability, Health Information Interoperability/standards, Health Planning Technical Assistance, Planning Techniques, IHE, Integrating the Healthcare Enterprise, $3 \mathrm{LGM}^{2}$, Modeling Information System Architecture

\section{Introduction}

\subsection{Background}

There are many specialized application systems in healthcare and often they require the same information. If possible, this information should only be collected once and then exchanged between the application systems. Therefore, communication standards have

${ }^{1}$ Corresponding Author: Angela Merzweiler, Department MIS, Universitätsklinikum Heidelberg, Im Neuenheimer Feld 130.3, 69120 Heidelberg, Germany; E-mail: angela.merzweiler@med.uni-heidelberg.de. 
been defined. Most syntactic standards have been defined so that they can be used for many use cases. Accordingly, they give users a lot of freedom. E.g., Health Level Seven ${ }^{2}$ version 2 (HL7v2) OBX segments can transmit all types of observations like laboratory values or metrics from medical devices. That is why, profiles for certain use cases are already defined within these standards (e.g. FHIR $^{3}$ profiles for family planning [1]), in order to restrict the use of the standard (e.g., binding of values to value sets of special code systems (e.g. LOINC ${ }^{4}$ Codes for laboratory values or IEEE $11073.10101^{5} \mathrm{Codes}$ for metrics of medical devices) in order to improve semantic interoperability. Over time, many different standards have been developed, which have their strength in certain use cases, e.g.: DICOM 6 for radiology systems, HL7v2 for the communication of clinical information systems or HL7 FHIR for mobile applications. Therefore, these standards are used in information systems in healthcare. In order to improve communication between application systems using the most appropriate standard, Integrating the Healthcare Enterprise (IHE) was founded in 1998 in the USA ${ }^{7}$. Since then, IHE International $^{8}$ has defined integration profiles that describe how information can be transported, how workflows should be implemented or what content should be exchanged between application systems. E.g., the Scheduled Workflow Profile, describes the correct interaction between an order placer, a radiology information system (RIS), various modalities and the picture archiving and communication system (PACS). Communication here is based partly on HL7v2 and partly on DICOM. The most important components of an integration profile are: actors (functional component of a system that exchanges transactions with other actors), transactions (set of messages exchanged by the actors) and the transported content (representation of information that is exchanged). Optional properties have been defined for some actors. These can extend the functionality of the actor (e.g., the actor can not only display the data but also import it). In addition, there are often rules within an integration profile. E.g., certain actor instances may only exist once (e.g. registry in the Cross Enterprise Document Sharing profile (XDS.b)) and others several times (repositories, consumers, sources) within one instance of a profile [2]. So, it is often required that actors have to be grouped with actors of other profiles, which means that they are part of the same application system.

Over the years, IHE International has defined more than 200 integration profiles ${ }^{9}$. Therefore, inexperienced modelers of information systems quickly lose track of existing IHE profiles. In addition, the users of these profiles are often not aware of rules that are defined within these profiles and of dependencies that exist between the profiles. There are also modelers that do not notice that the functionality of the same actor can differ between different application systems due to the range of their optional capabilities.

The aim of this paper is therefore to describe a concept how modelers of information systems can be supported in the selection and correct use of IHE profiles and how this

\footnotetext{
2 https://www.hl7.org/

${ }^{3}$ https://www.hl7.org/fhir/

${ }^{4}$ https://loinc.org/

${ }^{5}$ Point-of-care medical device communication, https://ieeexplore.ieee.org/document/8863790

${ }^{6}$ https://www.dicomstandard.org/

${ }^{7}$ https://www.ihe.net/about_ihe/faq/

${ }^{8}$ https://www.ihe.net/

${ }^{9}$ https://wiki.ihe.net/index.php/Profiles
} 
concept was prototypically implemented in the "Three-layer Graph-based meta model" modeling tool $\left(3 \mathrm{LGM}^{2} \mathrm{Tool}^{10}\right)$.

To increase the readability of this article, the following use case is consistently considered in this article: "A modeler wants to plan an electronic health record that is accessible by a hospital including the Laboratory Information System (LIS) and patients."

\subsection{Requirements}

Based on our previous requirements analysis [3], the modeler must be supported in:

- $\quad$ selection of suitable integration profiles

- selection of suitable actors (and their options) of the profile

- selection of suitable transactions

- proofing the necessary grouping with actors from other integration profiles

- support in checking the consistency of the model

\section{State of the art}

Enterprise architecture modelling tools like Zachman ${ }^{11}$ or $3 \mathrm{LGM}^{2}[4,5]$ provide modeling support and are able to answer questions about the modeled information system. However, until 2015 none of them provided direct support for the use of IHE profiles. Stäubert et al. have already designed a first approach in 2015 [6] and within the 3LGM2IHE project ${ }^{12}$ a refinement was made in 2019 [3] to close this gap. At this time, $3 \mathrm{LGM}^{2}$ tool was able to offer IHE profiles for modeling information systems. But the tool did not sufficiently support the user to find appropriate profiles. E.g., profiles could only be found searching for terms used within the names of the profiles. Unfortunately, this method is not sufficient to find all appropriate IHE profiles. E.g., if you search for profiles by the term "document", the profile Cross Community Access (XCA) was not found, even though it is used for querying documents from other IHE affinity domains. The tool was also not able to validate models whether they stick to restrictions, like correct cardinality of actors or required actor groupings. So, modeling of information systems using IHE profiles was quite uncomfortable and resulted sometimes in inconsistent models, when the user was not familiar with the restrictions defined within these profiles. That is why the user should be supported in the modeling process.

\section{Concept}

During the modeling process (cf. fig. 2), the users must accomplish the following steps: Step 1: First, users must precisely define the use case for which they plan the (part of) information system. E.g.: Document exchange via a commonly used electronic health record (EHR). The best way to support the modeler is to provide a hierarchy of use cases

\footnotetext{
${ }^{10} \mathrm{http}: / / \mathrm{www} .3 \operatorname{lgm} 2 . \mathrm{de} / \mathrm{en} /$

11 https://zachman.com/

12 https://gepris.dfg.de/gepris/projekt/315068407?language=en
} 
to select the right use case. Alternatively, modelers can also search for the use case using keywords, but this is often not sufficient.

Step 2: By selecting the use case, the selection of suitable integration profiles is already severely restricted. In our case, only the XDS.b profile can be used for EHR based exchange of patient documents. However, this could be enhanced by the Mobile access to Health Documents profile (MHD) for the provision of patient reported outcomes or current medication plans for patients, since this is better suited for communication with mobile devices [7]. Similarly, for other use cases (e.g. management of IDs), users can choose between comparable profiles based on different standards.

Figure 1 shows the selection of use cases and the corresponding integration profiles. (The selection of the integration profiles can be further restricted by a more detailed description of use cases.)

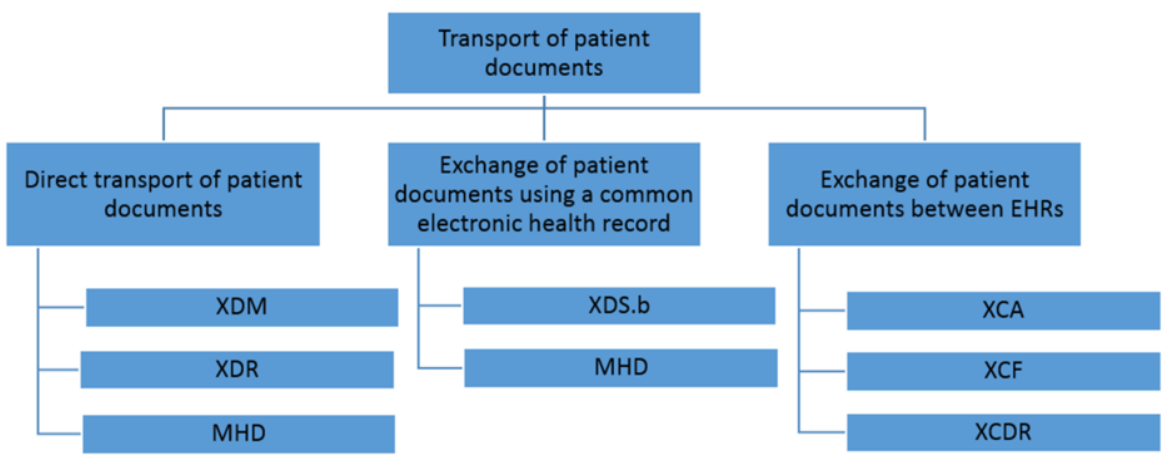

figure 1. This decision tree shows three types of use cases where patient documents are transported. Each subtype restricts the set of appropriate IHE integration profiles shown at level three.

Step 3: In a third step, modelers have to choose which and how many actors of the integration profile they need. The profile often specifies the number of actors that may be instantiated in a cluster of application systems. E.g., an Affinity Domain may only have one registry. If modelers plan several registries, they must be informed that this is not permitted. However, they can plan one or more repositories and as many XDS.b document sources and consumers as required. In some cases, individual actors can also be left out entirely, e.g. an OnDemand Document Source since this is an optional actor of XDS.b. If MHD Document Sources and Document Consumer should be used to connect the mobile devices to the EHR, they have to be grouped with specific actors from the XDS.b profile as this grouping is required to communicate with an XDS.b infrastructure. That's why users should be informed about required groupings. The demand of these groupings can be cascaded. For example, each XDS.b actor must be grouped with an Audit Trail and Node Authentication (ATNA) secure client or secure application. Each ATNA actor must in turn be grouped with a CT Time Client so that the time synchronization of the systems is guaranteed. After adding an ATNA secure application system, users must also be informed that they have to add further actors of the ATNA integration profile as an ATNA repository must be added in which the log messages can be saved. Each instance of an actor must also be assigned to exactly one application system. If this rule is not accomplished, modelers have to be warned. On the other hand, an application system can include several actor instances of different actors 
because of the required actor groupings or in order to provide an expanded range of functions.

Step 4: After actors were added, the required functionality of the instantiated actors must be further specified. E.g., not all instantiations of XDS.b Document Sources support the option of storing documents in folders in a patient record. Only some instantiated actors support all specified transactions. It is therefore important that not only the actor but also the necessary or actually supported options of this instantiation can be modeled in an application system. By selecting an option, further constraints can be activated that require further actor groupings. E.g., by selecting the folder option mentioned above, the specification informs that the document source may be grouped with a document consumer ${ }^{13}$. Modelers must also be informed about this.

Step 5: Modeling the transactions: Once all actors (including options) have been selected, the possible communication connections have to be added. E.g., it must be determined: which document source instance can upload documents to which repository instances. When connecting the patient identity source to the registry, the interface of the registry that supports the same transaction as the patient identity source must be connected. E.g.: The registry must be connected to a patient identity source either via an ITI-8 or an ITI-44 transaction. If an application system includes the functional scope of several actors (e.g. repository and registry), who normally communicate with one another using a transaction (e.g. ITI-42), the application system can, but does not have to, provide a corresponding transaction interface for other application systems.

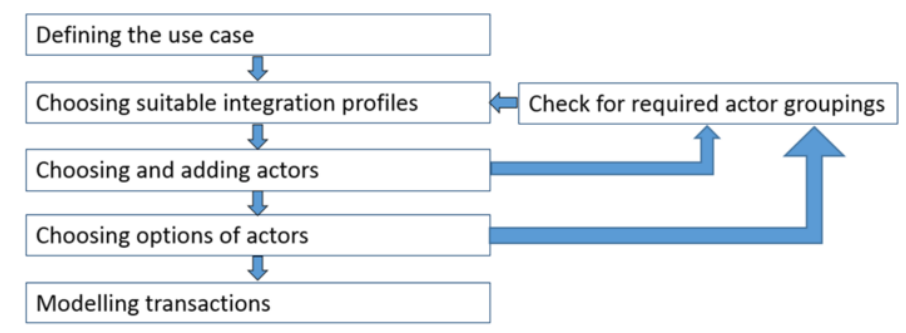

figure 2. The modeling process with possible loops

\section{Implementation}

The following section describes how the modeling process (see chapter 3 ) is supported by the new implemented functions of the $3 \mathrm{LGM}^{2}$ Tool from the perspective of the modelers.

To support step 1 (defining the use case) and step 2 (choosing suitable integration profiles) we implemented the template browser. The IHE elements are presented in a hierarchy where modelers can browse them. To get more information about a certain IHE concept, e.g. an actor, modelers can go to right-click $\rightarrow$ Properties (see Fig.3, no.2). Depending on the type of element selected, a description, an overview and separate tabs with related actors or transactions are presented.

To support step 3 (choosing and adding actors; check for required actor grouping) modelers can go to right-click $\rightarrow$ 'Add To Model'. Groupings are taken into account and

${ }^{13}$ https://www.ihe.net/uploadedFiles/Documents/ITI/

IHE_ITI_TF_Vol1.pdf\#nameddest=10_2_4_Folder_Management_Option 
the actors to be grouped are also included in the model. Now the added actor and all actors that need to be grouped with him are available in the model and appear in the model browser on the left (ref. Fig 3, no.3). Then instances of this actor can be created and assigned to an application system. The assignment of exactly one actor instance to exactly one application system is ensured by corresponding restrictions in the underlying metamodel. As a result, the actor instance is created, assigned to the application system and displayed as a hexagon in the model graph (ref. Fig.3, no.4). If groupings are subsequently required, this is detected by the consistency check of the model and a corresponding message is displayed (ref. Fig.3, no.5). A tool tip informs users which actors are available for a grouping (ref. Fig.3, no.6). To fix this consistency issue, the modeler can right-click $\rightarrow$ connect on the message and select the actor(s) to be grouped. This will create instances of these actors and add them to the application system affected. In our example, the problem was solved by adding (grouping) the actor Secure Application from the ATNA profile to the XDS.b Document Source actor in the LIS application system. The Secure Application actor in turn requires grouping with the Time Client from the Consistent Time (CT) profile. With these additions, a cascade of actors to be grouped starting from the XDS.b document source for the LIS application system was taken into account (ref. Fig.3, no.7).

Step 4 (choosing options of actors) can also be supported in the future by asking the modeler, when instantiating an actor, which options should be selected for the actor instance. The dependencies resulting from the combination of options can be identified by the consistency check and then resolved.

To support step 5 (modeling transactions), the $3 \mathrm{LGM}^{2}$ Tool supports the modeler by highlighting possible target IHE interfaces when selecting an IHE interface (same transaction must be supported).

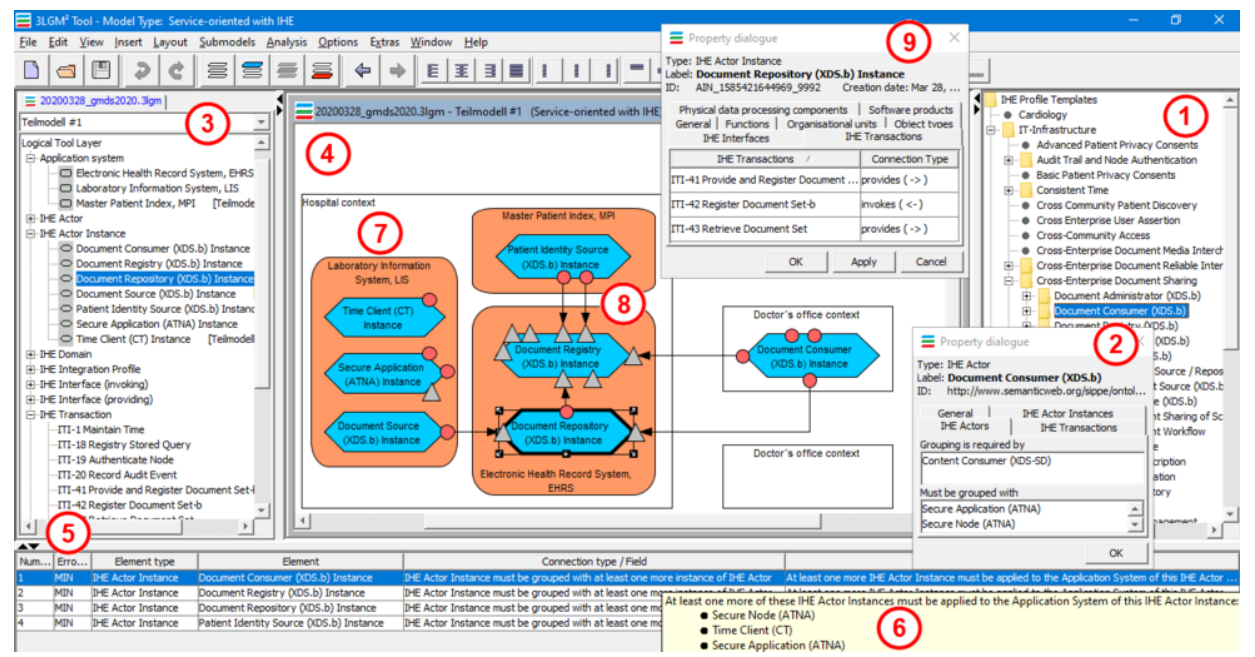

figure 3. Screenshot of the $3 L G M^{2}$ Tool. 1) Template Browser with hierarchy of IHE template elements; 2) IHE Actor property dialog with 'must be grouped with' relation; 3) Model browser with hierarchy of model elements; 4) model graph view with depicted 7) application systems (rounded rectangles) and IHE actor instances (hexagons), invoking interfaces (circles) can initiate communication via 8) communication links (directed edges between interfaces) to providing interfaces (triangles), IHE transactions (not displayed) are associated with interfaces and communication links; 9) IHE Actor Instance property dialog of the selected (strong) XDS.b Document Repository actor with transactions; 5) issues from consistency checks; 6) tooltips on an issue that helps to solve the issue 
In order to make the $3 \mathrm{LGM}^{2}$ Tool easier to maintain, we created the IHE knowledge base as an ontology by using protegé [8]. The ontology includes information about the following IHE concepts: domain, integration profile, actor, transaction and how they interact with each other (communication, grouping, etc.) including options and restrictions. The ontology is used in the $3 \mathrm{LGM}^{2}$ Tool, e.g. to provide these information in the template browser (see Fig.3, no.1). A detailed description of the software architecture of the modified $3 \mathrm{LGM}^{2}$ tool will be presented in a future paper.

\section{Lessons learned (Discussion)}

Our approach addresses all requirements: it supports the user in the selection of suitable integration profiles, actors (and their options) of the profile, transactions, proofing the necessary grouping with actors from other integration profiles and in checking the consistency of the model. But the implementation has still some limitations:

- search is currently only supported within the model but not in the templates

- Cardinality restrictions between actors are not yet considered when establishing models, but they are modeled in the underlying ontology.

- Options of actors cannot be modeled at this time. Therefore, required actor groupings resulting from the selection of an option cannot be verified.

Another tool that implements any kind of support could not be found via literature research. The tool should support the entire described modeling workflow. Due to the complexity and available project resources, the usability should be optimized to achieve a user experience comparable to other modeling tools, which has been postponed to a later stage.

\section{Conclusion}

"The future [of EHRs] lies in the development of flexible component-based architectures that can operate seamlessly within the workflow of a healthcare environment." [9](p.54) Therefore EHRs are expected to be based on internationally accepted standards in the next 25 years [9]. To match such needs, IHE offers the needed integration profiles. But modeling IHE compliant information system architectures is challenging. Many rules that are described in free text in the IHE Technical Frameworks ${ }^{14}$ have to be transformed into complex rules that can be processed by computers. However, there are also other aspects of modeling, such as access control mechanisms, which are not described in the IHE profiles ( except BPPC, APPC consent profiles), which we have not discussed. The metamodel underlying this work is based on the 3LGM2-S [10]. We plan to publish an updated version of the metamodel when it is finalized. Further implementation effort and evaluation of our approach by more users are needed. It is expected, that this tool will become more valuable tool for modelers planning healthcare information system architectures, in particular those based on IHE. 3LGM ${ }^{2}$ Tool is available for download on the $3 \mathrm{LGM}^{2}$ homepage $^{15}$ and via TMF ToolPool Gesundheitsforschung ${ }^{16}$. The model

\footnotetext{
14 https://www.ihe.net/resources/technical_frameworks/

15 http://www.3lgm2.de/en/Downloads/3LGM2_Tool/index.jsp (please use version 4.2 or above)

16 https://www.toolpool-gesundheitsforschung.de/produkte/3lgm2-baukasten
} 
used in this article can be found in the $3 \mathrm{LGM}^{2}$ Tool (since Version $4.2 \mathrm{RC}$ ) under Help $\rightarrow$ 'Model Library...': IHE\gmds2020_IHE_Grouping-Example.3lgm.

\section{Conflict of Interest}

The authors state that they have no conflict of interests according to the ICJME principles.

\section{Acknowledgements}

This work was funded by the German Research Foundation (DFG), project number: 315068407. This work was conducted using the Protégé resource, which is supported by grant GM10331601 from the National Institute of General Medical Sciences of the United States National Institutes of Health.

\section{References}

[1] Matney SA, Heale B, Hasley S, Decker E, Frederiksen B, Davis N et al. Lessons Learned in Creating Interoperable Fast Healthcare Interoperability Resources Profiles for Large-Scale Public Health Programs. Appl Clin Inf 2019; 10(1):87-95.

[2] Integrating the Healthcare Enterprise (IHE). IT Infrastructure Technical Framework: Volume 1 (ITI TF-1) Integration Profiles; Revision 16.0; 2019 Jul 12 [cited 2020 Apr 3]. Available from: URL: http://www.ihe.net/uploadedFiles/Documents/ITI/IHE_ITI_TF_Vol1.pdf.

[3] Stäubert S, Strübing A, Drepper J et al. Towards a Software Tool for Planning IHE-Compliant Information Systems. Stud Health Technol Inform 2019; 258:6-10.

[4] Winter A et al. Modeling hospital information systems (Part 1): The revised three-layer graph-based meta model 3LGM2. Methods Inf Med 2003; 42(5):544-51.

[5] Wendt T et al. Modeling Hospital Information Systems (Part 2): using the 3LGM2 tool for modeling patient record management. Meth Inf Med 2004; 43(3):256-67.

[6] Stäubert S, Schaaf M, Jahn F, Brandner R, Winter A. Modeling Interoperable Information Systems with 3LGM² and IHE. Meth Inf Med 2015; 54(5):398-405.

[7] IT Infrastructure Technical Framework Technical Framework Supplement: Mobile access to Health Documents (MHD) With XDS on FHIR; Revision 3.1 - Trial Implementation; 2019 Mar 6 [cited 2020 Apr 3]. Available from: URL: https://www.ihe.net/uploadedFiles/Documents/ITI/IHE_ITI_Suppl_ MHD.pdf.

[8] Musen MA. The Protégé Project: A Look Back and a Look Forward. AI Matters 2015; 1(4):4-12.

[9] Evans RS. Electronic Health Records: Then, Now, and in the Future. Yearb Med Inform 2016; Suppl 1:S4861.

[10] Winter A, Ißler L, Jahn F, Strübing A, Wendt T. Das Drei-Ebenen-Metamodell für die Modellierung und Beschreibung von Informationssystemen (3LGM² V3): Universität Leipzig; 2010. IMISE Reports 1/2010 [cited 2020 Jul 17]. Available from: URL: http://www.3lgm2.de/Publikationen/Dokumente/3LGM_V3 IMISE-Report_final.pdf. 